\title{
Effects of non-grey radiative transfer on 3D simulations of solar magneto-convection
}

\author{
A. Vögler ${ }^{\star}$ \\ Max-Planck-Institut für Sonnensystemforschung ${ }^{\star \star}$, Max-Planck-Strasse 2, 37191 Katlenburg-Lindau, Germany \\ Received 9 January 2004 / Accepted 24 March 2004

\begin{abstract}
We compare 3D simulations of photospheric magneto-convection which include a multigroup modelling of the non-grey radiative transfer with simulations based on a grey radiative transfer using the Rosseland mean opacity. While the simulation properties in the solar surface layers around $\tau_{\text {Ross }}=1$ are very similar both cases, pronounced differences occur both in the temperature structure of magnetic field concentrations in the upper photosphere and in the appearance of strong magnetic fields in brightness maps. The results show that a non-grey treatment of the radiative transfer is mandatory if magnetoconvective simulations are to be compared with observational results by calculating synthetic spectra of temperature-sensitive
\end{abstract} \\ lines originating in the middle and upper photosphere.
}

Key words. magnetohydrodynamics (MHD) - Sun: magnetic fields - Sun: photosphere

\section{Introduction}

The photosphere is the layer within the solar atmosphere where the transition from mainly convective to radiative energy transport occurs. Since the radiation field plays a crucial role in the overall energy balance of the photosphere and since radiative cooling in the photospheric layers acts as the main driver of convection, numerical simulations aiming at a realistic description of the photospheric conditions must include a detailed treatment of radiative transfer effects. It is well known that the inclusion of line opacities in calculations of stellar model atmospheres strongly modifies the resulting teperature profiles, leading to considerably cooler outer layers, while the temperature is raised in deeper regions (the line cooling and backwarming effects, see e.g. Mihalas 1978). Therefore, the numerical treatment of radiative transfer in simulations should account for the effects of line opacities.

The opacity in the solar atmosphere includes the contributions of about $10^{6}$ atomic and molecular spectral lines, so that, in principle, $10^{6}-10^{7}$ frequency points are necessary to cover its detailed frequency dependence. In time-dependent threedimensional simulations the computational costs of such a direct approach are intolerable and a statistical description of the effects of a frequency-dependent opacity is necessary in order to drastically reduce the computational expense. Among the strategies devised to tackle this problem are the method of opacity sampling (see, e.g., Sneden et al. 1976), the concept of Opacity Distribution Functions (ODF, Strom \& Kurucz 1966; Mihalas 1967), and the multigroup (or opacity binning)

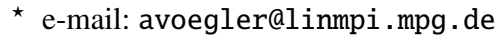

$\star \star$ Formerly: Max-Planck-Institut für Aeronomie.
}

method (Nordlund 1982; Ludwig 1992; Ludwig et al. 1994; Vögler et al. 2003). In the context of time-dependent threedimensional simulations, for which the radiative transfer must be solved for every timestep, the method of choice - given the currently available computing resources - is the multigroup method since it yields the most drastic reduction of computational cost and allows for a satisfactory approximation of the frequency-dependence with only a few representative frequency bins.

However, even when the multigroup approach is used, the non-grey treatment of the radiative transfer increases the computational costs of a simulation significantly, and typically 80-90\% of the computing time is spent in calculating the radiative transfer. This imposes serious restrictions on the size of numerical simulations, and many interesting applications, which require large computational domains, high spatial resolution or a long simulated time span to be covered, can currently not be carried out with a non-grey radiative transfer. Therefore it seems worthwhile to study systematically how strongly the properties of a photospheric MHD simulation are affected by the non-grey treatment, and possibly identify those cases which can be satisfactorily simulated with a grey radiative transfer. To this end, we analyze 3D MHD simulations of a solar plage region which were carried out with the MURaM code ${ }^{1}$ (Vögler 2003; Vögler et al. 2003). In order to isolate the effects of the frequency-dependent radiative transfer, we compare two simulation runs with identical setup and choice of parameters, except for the radiative transfer part. One simulation run includes

\footnotetext{
1 The MPAe UofC Radiation Magneto-hydrodynamics code resulted from a cooperation of the solar MHD groups at the Max-Planck-Institut für Aeronomie and the University of Chicago.
} 
a non-grey treatment based on the multigroup method with four opacity bins, the other run uses the grey approximation with a standard Rosseland mean opacity. Section 2 gives a brief description of the numerical methods and the simulation setup, Sects. 3 and 4 discuss similarities and differences between the two simulations and Sect. 5 presents the conclusions.

\section{Description of the numerical model}

The MHD simulations of photospheric magnetoconvection on which this study is based were carried out with the MURaM code, which solves the time-dependent MHD equations for a compressible and partially ionized plasma with radiative transfer. The radiative transfer equation is treated under the assumption of local thermodynamic equilibrium. The bottom boundary condition allows for free in- and outflow of matter, maintaining a constant total mass in the box as well as a fixed energy flux through the system, while the top boundary is closed. The magnetic field is assumed to be vertical at the top and bottom boundaries, the footpoints of fieldlines are allowed to move freely. The horizontal directions are taken to be periodic.

The simulation setup for the grey and the non-grey simulation runs was identical: the dimensions of the computational domain are $1400 \mathrm{~km}$ in the vertical direction and $6000 \mathrm{~km}$ in both horizontal directions, with a resolution of $100 \times 288 \times$ 288 grid points. The simulations started from a plane-parallel model of the solar atmosphere extending from $800 \mathrm{~km}$ below to $600 \mathrm{~km}$ above the level of continuum optical depth unity at $500 \mathrm{~nm}$, which is chosen as the zero-level of the height coordinate $z$ used in the following sections. After convection had fully developed, a homogeneous vertical initial field of 200 Gauss was introduced. Within a few minutes of simulated time (approximately one turnover time of the convection) most of the magnetic flux is swept into the intergranular downflow lanes. Larger magnetic structures (micropores) form at granule vertices where several downflow lanes merge. A detailed description of the code and a discussion of some results of magnetoconvection simulations are given in Vögler et al. (2003), Vögler \& Schüssler (2003), Schüssler (2003) and Vögler (2003).

For the grey simulation run, the Rosseland mean opacity was used. In case of the non-grey simulation run, the multigroup method with four opacity bins was used to account for the frequency dependence. The basic idea of the multigroup method is to divide the frequency spectrum into a small number of (non-contiguous) bins based on the height in a reference atmosphere where $\tau_{v}=1$ is reached (Nordlund 1982). Frequencies which reach $\tau_{v}=1$ at a similar height are collected in the same bin. For each bin, a bin-averaged opacity table is calculated and the radiative transfer equation for the bin-integrated intensity is solved. Frequency-integrated quantities of the radiation field like the radiative energy flux or mean intensity, which are required in order to calculate the total radiative heating rate, are obtained by summing over the contributions from the different bins. See Vögler et al. (2004) for details regarding the multi-group implementation used here. In both simulation runs, the radiation field has an angular resolution of 24 directions with 3 directions per octant.

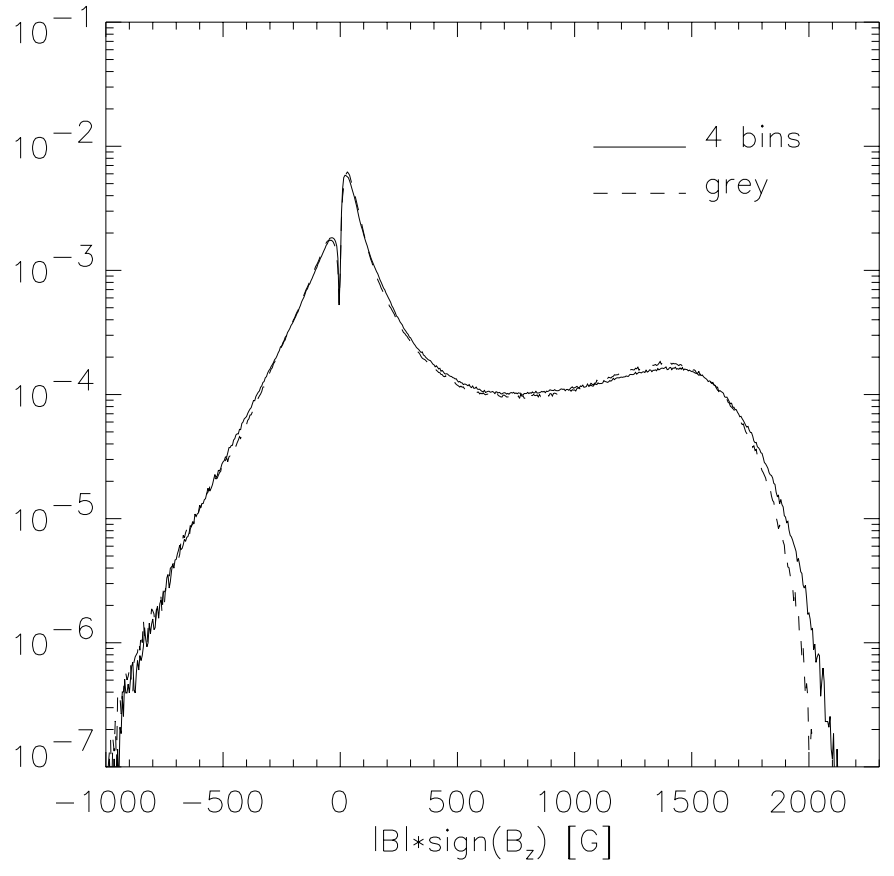

Fig. 1. Probability distribution function of the magnetic field strength in a layer of $100 \mathrm{~km}$ thickness around $\left\langle\tau_{\text {Ross }}\right\rangle=1$, multiplied with the sign of the vertical field component. In the non-grey case, the strong field component is shifted slightly towards higher field strengths. Otherwise, the distributions are identical.

\section{Simulation properties near $\tau_{\text {Ross }}=1$}

When comparing the two simulations, it turns out that many properties in the surface layer around $\tau_{\text {Ross }}=1$ (and below) are similar. Once the system has relaxed to a statistically stationary state (1-2 turnover times after the introduction of the magnetic field), the magnetic field in both cases is organized in a network structure outlined by the network of intergranular downflows, with thin sheet-like structures stretching along the downflow lanes and micropores occasionally forming at granular vertices. Morphology and time evolution of the magnetic field and the granular pattern are very similar. This is plausible since the structure of the magnetic field is mainly determined by its interaction with the convective flows in the layers around and below the surface, where the differences between the two radiative transfer models are marginal. In both cases the radiative cooling at the surface level around $\tau_{\text {Ross }}=1$ acts as the main driver of convection and the cooling layer is in both models sharply defined by the steep increase of the opacity with temperature. The total cooling rate is determined by the total energy flux and is independent of the treatment of the frequency dependence of the radiative transfer. Consequently, the convective flow fields are similar. At this height level, systematic differences in the statistical properties of the two simulations are very small. As an example, Fig. 1 shows the probability distribution function (PDF) of the magnetic field strength in a layer of $100 \mathrm{~km}$ thickness around $\tau_{\text {Ross }}=1$. In both cases the PDF is characterized by an exponential distribution of weak fields and an approximately gaussian strong-field component. In the non-grey case the Gaussian "bulge" is somewhat shifted towards higher field strengths, indicating that the mechanism of 

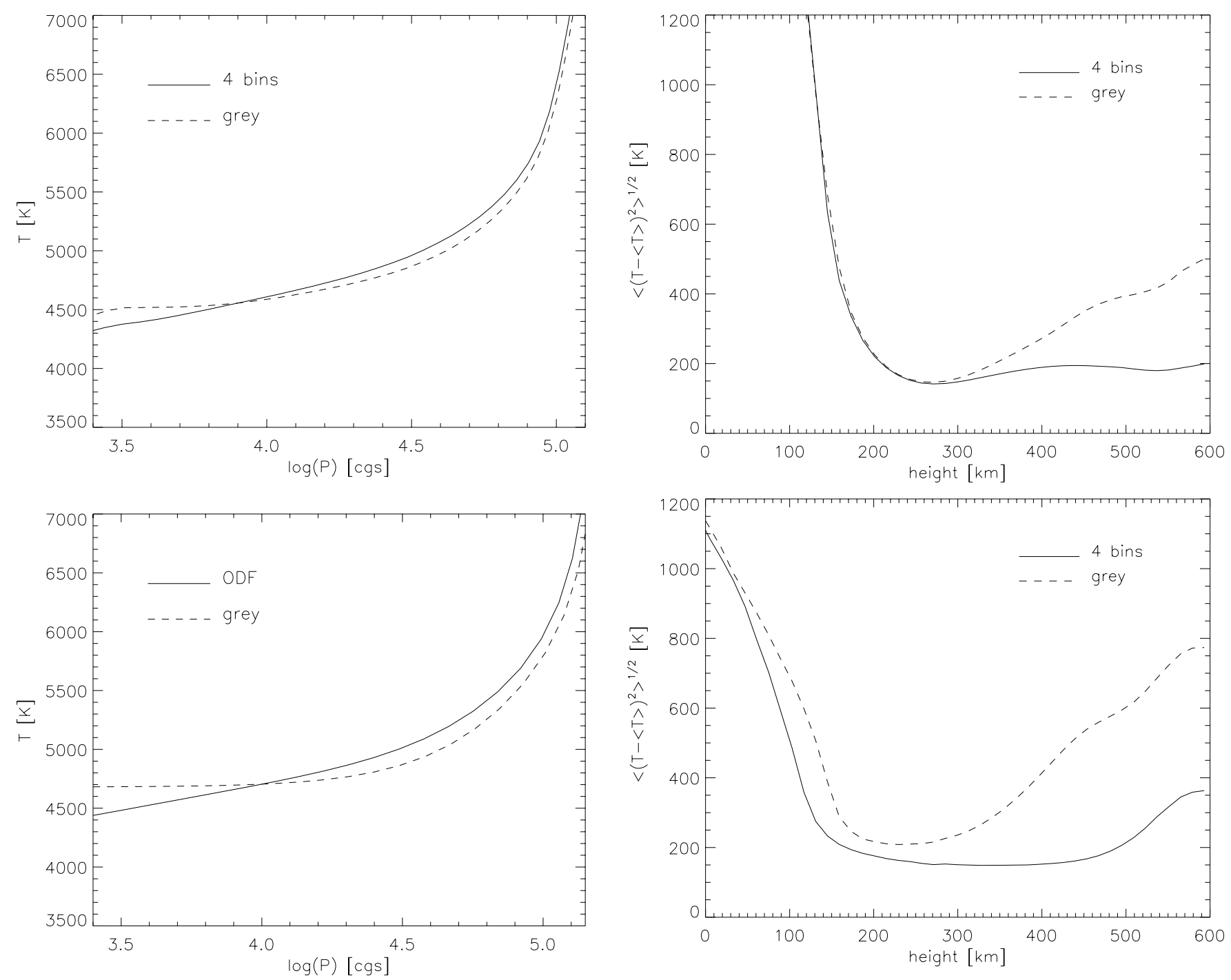

Fig. 2. Upper panel: horizontally averaged vertical temperature profiles for the 200-G simulation runs with grey and four-bin radiative transfer, respectively. Lower panel: temperature profiles of planeparallel solar model atmospheres obtained from the ATLAS9 code (Kurucz 1993), using Rosseland opacities and Opacity Distribution Functions, respectively.

convective field-amplification is slightly more effective. Apart from that, the two PDFs are practically identical. Consequently, important properties of the magnetic field at surface level, like its spatial structure, time evolution, and field strength distribution can be adequately modelled with grey radiative transfer calculations.

\section{Brightness and thermal structure in the upper photosphere}

\subsection{Average temperature structure}

Significant differences between the two simulation runs are expected to occur in the upper part of the computational domain above $\tau_{\text {Ross }}=1$, where the frequency bins corresponding to line opacities contribute strongly to the radiative heating rate. As mentioned in Sect. 1, the essential non-grey effects

Fig. 3. Vertical profiles of the horizontally averaged temperature fluctuations for the grey and non-grey simulation runs in the height range above $z=0$. Upper panel: fluctuations in weak-field regions with $|\boldsymbol{B}|<250 \mathrm{G}$; lower panel: same in strong-field regions with $|\boldsymbol{B}|>$ $250 \mathrm{G}$. The non-grey radiative energy exchange strongly reduces temperature fluctuations in the upper layers of the computational domain, particularly so in regions of strong magnetic field.

in plane-parallel model atmospheres are the "line-cooling" and "backwarming" effects. Figure 2 shows the horizontally averaged temperature profiles of the grey and non-grey simulation runs (upper panel) and the corresponding profiles of planeparallel atmospheres obtained with the ATLAS9 stellar atmosphere code (Kurucz 1993, lower panel) for comparison. The simulations show the same qualitative difference due to the non-grey treatment as the 1D models, the transition from backwarming to line-cooling in both cases occuring at the height level corresponding to $p=10^{4} \mathrm{dyn}_{\mathrm{cm}^{-2}}$.

\subsection{Temperature fluctuations in the upper photosphere}

Figure 3 shows that the non-grey treatment strongly affects the temperature fluctuations in the middle and upper parts of the 


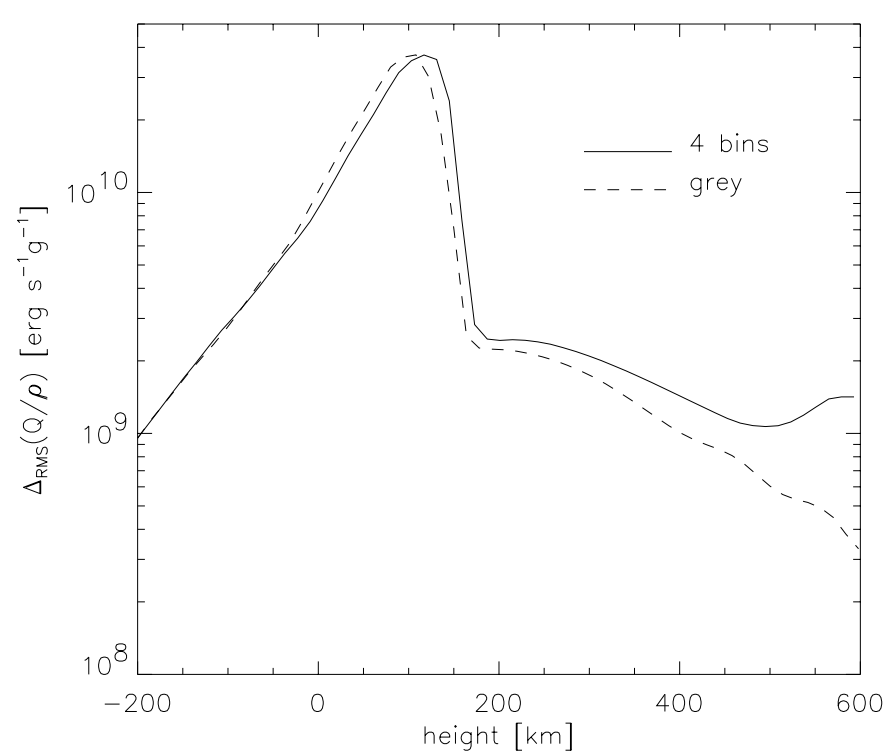

Fig. 4. Horizontal rms fluctuations of the radiative heating rate per mass unit, $Q / \varrho$, as a function of height. While the total radiative energy exchange in the upper photosphere is small for both models, the stronger fluctuations of $Q / \varrho$ in the non-grey case show the enhanced coupling between gas and radiation field, which tends to smooth temperature fluctuations.

photosphere. For the grey case, the rms temperature fluctuations on a given geometrical height level increase significantly with height above $z=300 \mathrm{~km}$; in magnetic flux concentrations (lower panel) the effect is more pronounced than in less magnetized regions (upper panel). The fluctuations of the nongrey case remain more or less constant for $z \geq 300 \mathrm{~km}$, with a slight increase in more strongly magnetized regions above $z=500 \mathrm{~km}$.

The difference between the two models results from the fact that the Rosseland mean underestimates the effect of line opacities, resulting in radiative energy exchange rates which are much too small in the optically thin parts of the photosphere. The opacity binning yields a much more adequate representation of line opacities. In this case, radiation field and plasma are more strongly coupled in the upper parts of the simulation box and radiative energy exchange smoothes temperature fluctuations more efficiently. The result that the fluctuations in the grey case are stronger inside magnetic flux concentrations than in weak field regions is due to the partial evacuation of strong fields which further reduces the radiative energy exchange. The difference regarding the coupling of the gas with the radiation field is quantified in Fig. 4. Fluctuations of the radiative heating rate around its mean value are significantly larger in the non-grey case, the excess over the fluctuations in the grey case increasing with height. The scatter diagrams in Fig. 5 show the relation between temperature and radiative heating rate in magnetic field concentrations at a height of $z=500 \mathrm{~km}$ above $\tau_{\text {Ross }}=1$. In both cases, $T$ and $Q_{\text {rad }}$ are correlated: hot regions are cooled, cool regions heated, so that in both cases the radiation tends to smooth out temperature fluctuations. The most significant difference between the grey (upper panel) and nongrey case (lower panel) can be seen in the low-temperature
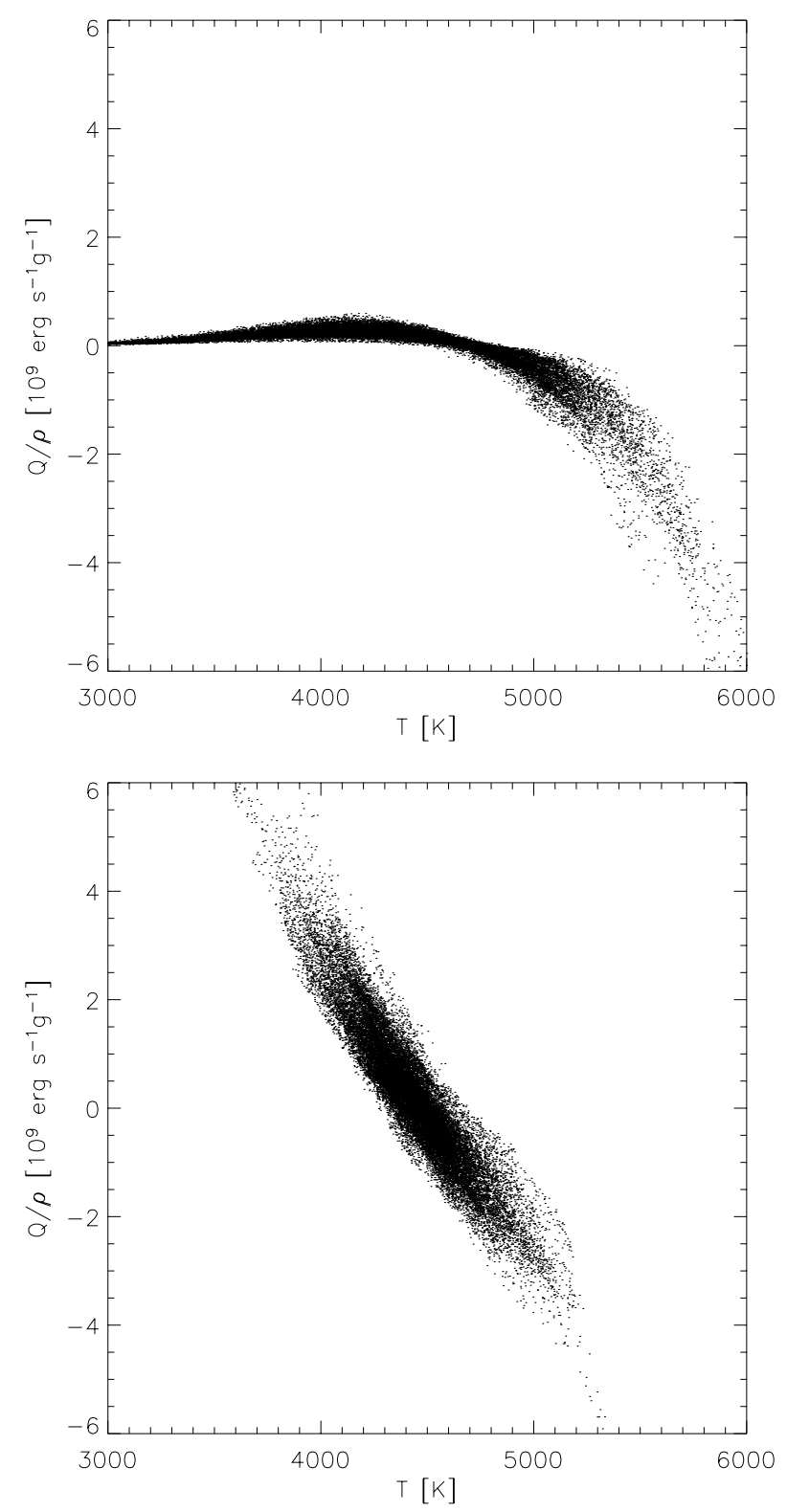

Fig. 5. Scatter plots of temperature versus radiative heating rate per mass unit, $Q / \varrho$, inside magnetic structures with $B_{z}>250 \mathrm{G}$, at a height of $500 \mathrm{~km}$ above $z=0$. Upper panel: grey case; lower panel: non-grey case.

wings of the scatter diagrams. In the non-grey model, the local heating increases roughly linearly with decreasing temperatures, the coolest places are those wich experience the strongest heating. In the grey case, the maximum values of $Q / \varrho$ are much smaller and the heating rates go to zero as temperatures become low. This can be understood as a consequence of the strong temperature dependence of the Rosseland opacity. For temperatures around and below $4000 \mathrm{~K}$, $\kappa_{\text {Ross }}$ falls off steeply with decreasing temperature, the correspondingly weak absorption of "hot" radiation is insufficient to balance local cooling processes (like adiabatic cooling of expanding gas).

The increase of the temperature fluctuations inside magnetic concentrations above $z=500 \mathrm{~km}$ for the non-grey 

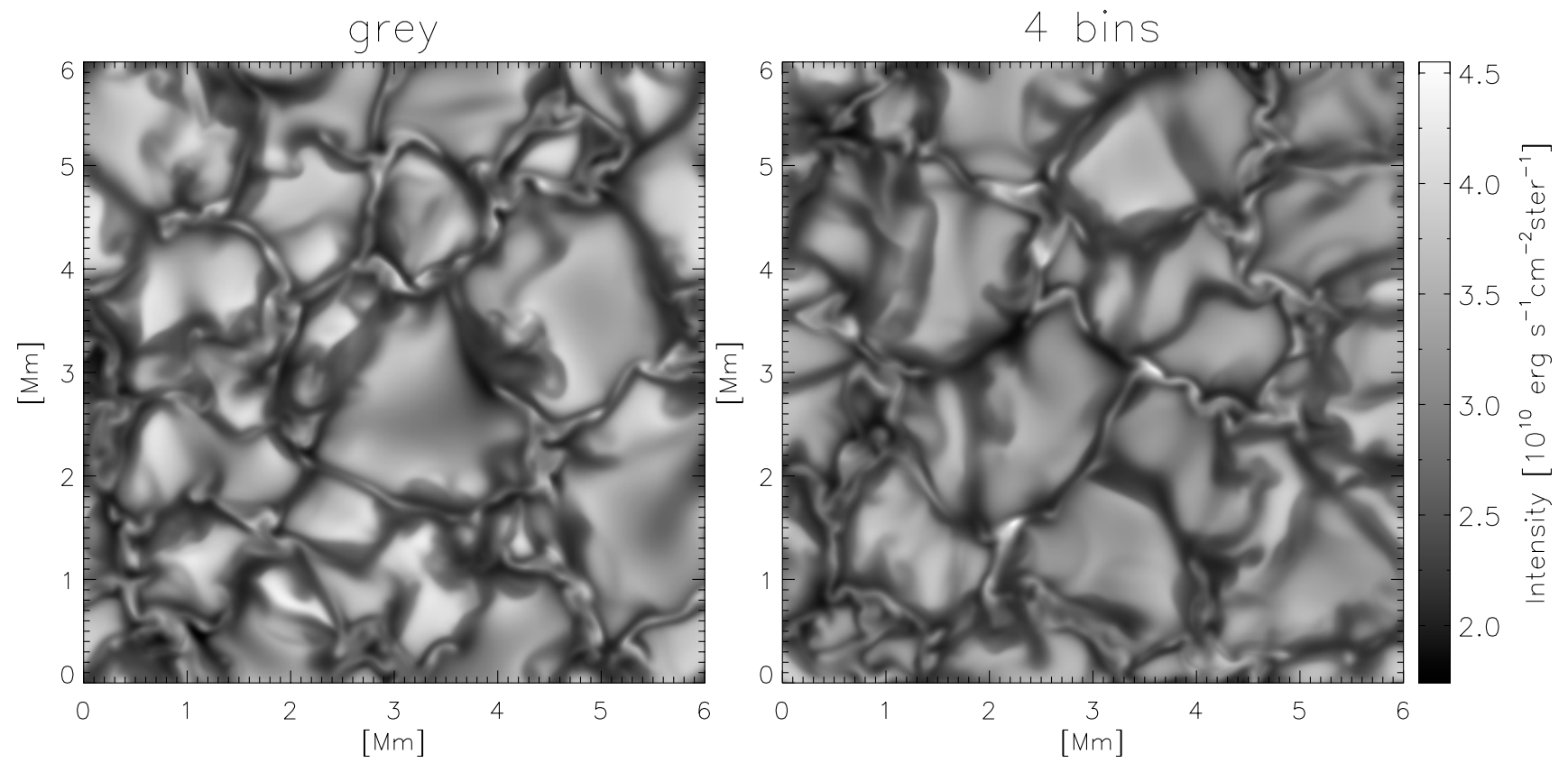

Fig. 6. Maps of frequency-integrated intensity for the grey (left panel) and non-grey (right panel) simulation runs, using the same greyscale coding for both images. In the non-grey case, granules are darker and the overall contrast is reduced. As a result, bright magnetic features stand out more prominently.

simulation run (lower panel of Fig. 3) is related to compression (expansion) effects which heat (cool) the gas locally. These are partly due to strong vortical flows which occur inside magnetic structures at that height, but could also be related to the closed upper boundary close to which any vertical gas motion inevitably involves compression/expansion effects. Furthermore, the $\tau=1$ level of the opacity bin representing the strongest line opacities - which is located near the top of the box in nonmagnetic regions - is significantly depressed inside magnetic flux concentrations. Therefore, those strong lines which reach $\tau=1$ in the partly evacuated magnetic regions near the top boundary, might not be well represented by the four-bin model. For this reason, the non-grey model tends to underestimate the radiative energy exchange there, which in turn contributes to the increase of temperature fluctuations.

\subsection{Brightness and intensity contrast}

It is plausible that the choice of the radiative transfer model should affect the appearance of the simulated structure in intensity pictures. The average rms intensity contrast (frequencyintegrated) of the four-bin simulation is reduced to 15 percent compared to the value of 18 percent for the grey run. As the comparison of brightness maps in Fig. 6 suggests, the lower contrast is mainly the result of a reduced brightness of granules $^{2}$. Since the intensities of the bright magnetic structures are largely unaffected, these features gain in contrast relative to the

\footnotetext{
${ }^{2}$ While the non-grey intensity map of vertical rays is clearly darker, this does not necessarily imply that less energy is radiated at the top of the box, since oblique rays also contribute to the total energy flux. In fact, in both simulation runs the same value for the radiative energy flux is obtained.
}

non-magnetic regions and thus stand out much more clearly against the granular pattern in the non-grey case. This impression from a single snapshot is corroborated by Fig. 7, which shows the joint probability distribution function (joint PDF) of intensity and magnetic field strength at $z=0$ for both runs, each based on one hour of simulated solar time. The greyscales indicate the probability of occurrence of a given value pair $(I, B)$ on a logarithmic scale. Owing to the smaller intensity variations in the weak-field part of the non-grey distribution (lower panel), the brightest magnetic features with field strengths around $2000 \mathrm{G}$ are brighter than the granules, while this is not true in the grey case (upper panel).

The result that the effect of non-grey radiative transfer on the intensity of granules is stronger than its effect on magnetic elements can be understood in terms of the respective temperatures at which the gas radiates. Figure 8 shows a scatter plot of temperature versus magnetic field strength on the $\tau_{\text {Ross }}=1$ isosurface for the grey run. In the non-grey case, the emerging intensity originates from four different height ranges defined by the respective $\tau=1$ levels for the four opacity bins. As can be seen from the corresponding scatter plots (Fig. 9), the lowest ("continuum") bin (BIN0, top left) radiates with a temperature distribution that is similar to the grey Rosseland case, except for a roughly uniform temperature increase of approximately $200 \mathrm{~K}$, which reflects the backwarming effect. The temperature distributions on the $\tau=1$ isosurfaces of the lineopacity bins (BIN1 to BIN3), however, show that the temperature at which the bright non-magnetic granules radiate drops drastically from $6900 \mathrm{~K}$ for BIN0 to $5000 \mathrm{~K}$ for the strongest bin (BIN3), summing up to a total intensity which is lower than in the grey case. Inside magnetic structures, however, the vertical temperature gradient is significantly smaller, so the 

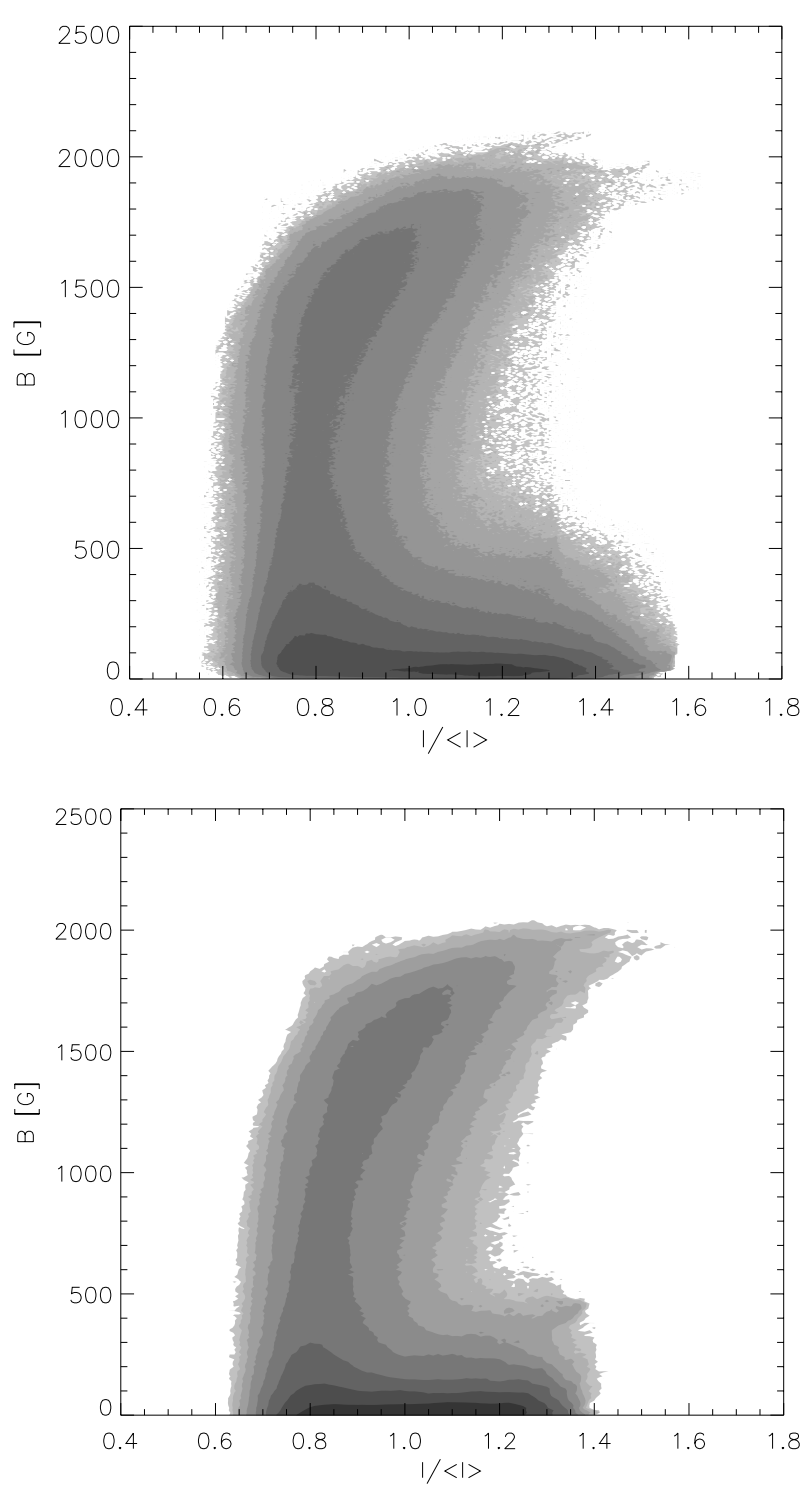

Fig. 7. Joint probability distribution functions showing the correlation between normalized emergent intensity and magnetic field strength at $z=0$. The greyscales indicate the probability of occurence of valuepairs $(I, B)$; the probability isolevels are spaced apart by 0.5 on the $\log _{10}$ scale. Upper panel: simulation run with grey radiative transfer. Lower panel: run with four opacity bins. The non-grey radiative transfer reduces the contrast of the nonmagnetic regions, thus enhancing the contrast of magnetic elements relative to the granules.

brightest magnetic elements radiate at much higher temperatures for the higher opacity bins. The resulting integrated intensity therefore exceeds that of the granules.

The increase in temperature in the upper layers of magnetic structures relative to non-magnetic regions at the same optical depth level is a result of heating by radiation from the hot bottom and walls of the magnetic flux concentrations. This "hot wall" effect has already been described by Spruit (1976, 1977) and was subsequently confirmed in two-dimensional simulations of magnetic flux sheets with grey radiative transfer (Deinzer et al. 1984; Knölker et al. 1991). Figure 10 illustrates the influence of the non-grey radiative transfer on the radiative illumination of magnetic flux concentrations in the simulation.

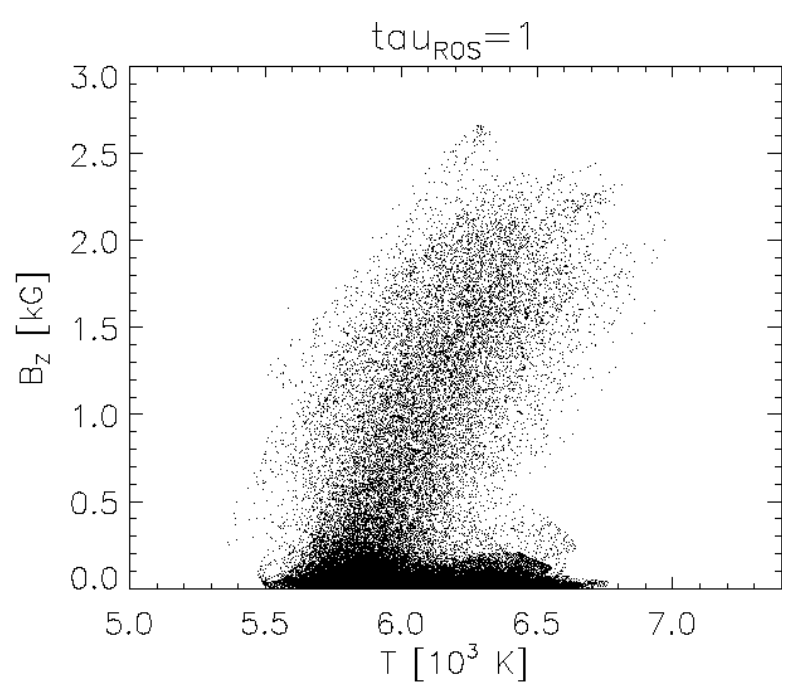

Fig. 8. Scatter plot of temperature vs. magnetic field strength on the isosurface $\tau_{\text {Ross }}=1$ for the simulation run with grey radiative transfer.

The solid lines show the mean vertical temperature profiles inside bright magnetic elements as function of the Rosseland optical depth; the mean profiles of the whole domain (dashed lines) are shown for comparison. In the optical depth range between $\log _{10} \tau_{\text {Ross }}=0$ and $\log _{10} \tau_{\text {Ross }}=-3$, the average temperature inside magnetic field concentrations of the non-grey simulation is clearly higher than in the grey case. The effect is most pronounced around $\log _{10} \tau_{\text {Ross }}=-1.5 \ldots-2$, where the temperature difference between the two cases reaches $200 \mathrm{~K}$. It is caused by the enhanced absorption of incident "hot" radiation in the layers above $\tau=1$ due to the line opacities accounted for in the non-grey model (i.e., the "backwarming" effect inside magnetic structures). As a result, the temperature increase of bright magnetic structures relative to the mean temperature profile (dashed lines) at $\log _{10} \tau_{\text {Ross }} \approx-2$ of approximately $400 \mathrm{~K}$ for the non-grey case is considerably larger than the corresponding temperature contrast of $250 \mathrm{~K}$ for the grey case. Therefore one expects that the non-grey radiative transfer will enhance the intensity contrast of bright magnetic elements relative to the average brightness in intensity maps obtained from the spectral line diagnostics of temperaturesensitive absorption lines originating at an optical depth of about $\log _{10} \tau_{\text {Ross }}=-2$.

\section{Conclusions}

The comparison between the grey simulation and the multigroup based non-grey simulation shows:

- The properties of the two simulations at the surface level around $\tau_{\text {Ross }}=1$ are very similar. Morphology, time evolution, and field strength distribution are largely the same.

- The average non-grey temperature profile differs from the grey profile in the optically thin part of the computational domain. It shows the backwarming and line cooling effects that are a familiar property of static 1D radiative equilibrium atmospheres.

- The use of a non-grey radiative transfer leads to a significant decrease of temperature fluctuations in the upper 

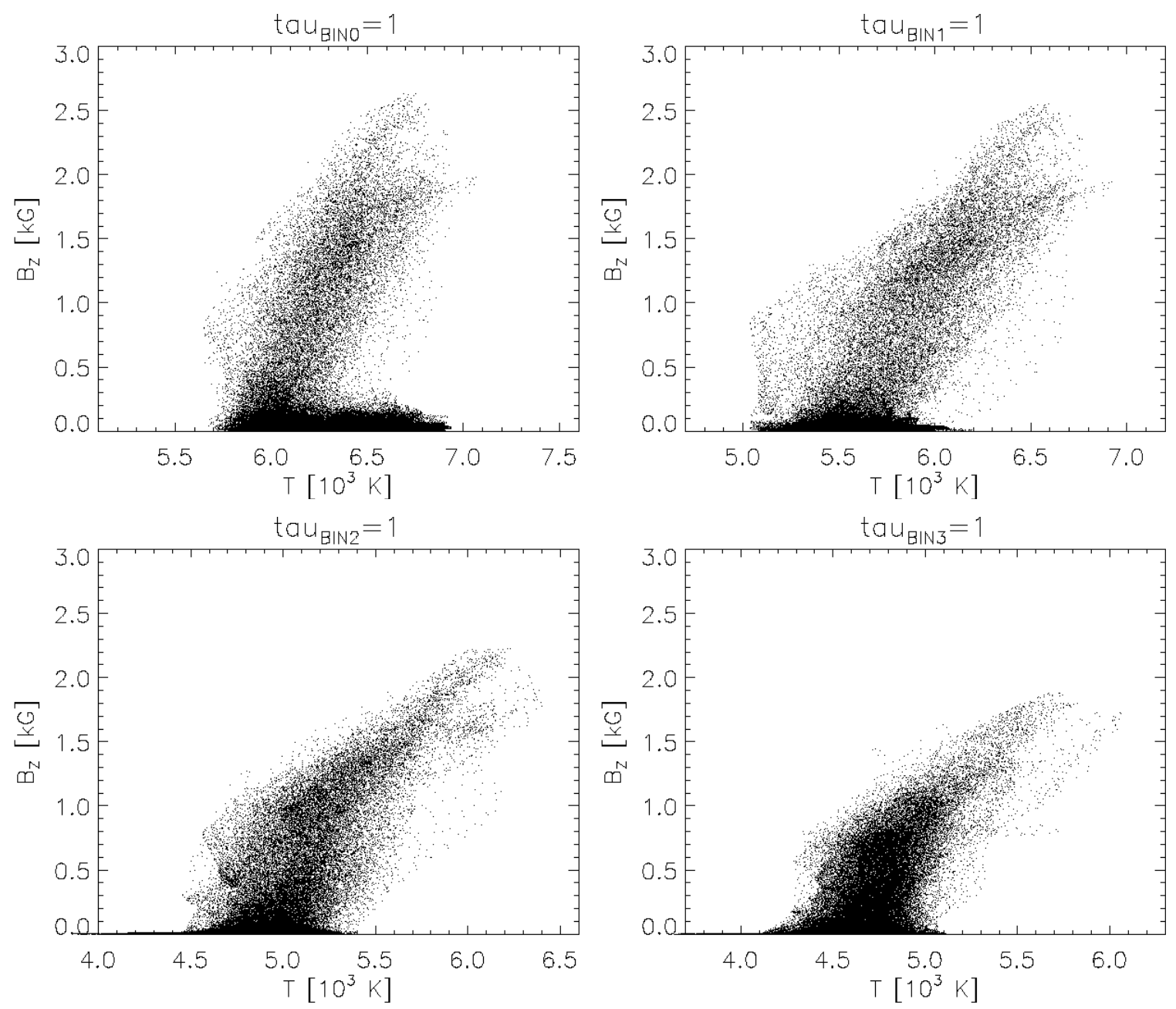

Fig. 9. Scatter plots of temperature versus magnetic field strength on $\tau=1$ isosurfaces for the four opacity bins of the non-grey simulation run. Top left: frequency bin representing continuum opacities ("BIN0"). The other three panels correspond to the line-opacity bins ("BIN1" to "BIN3", in ascending order of the height of the corresponding $\tau=1$ level.

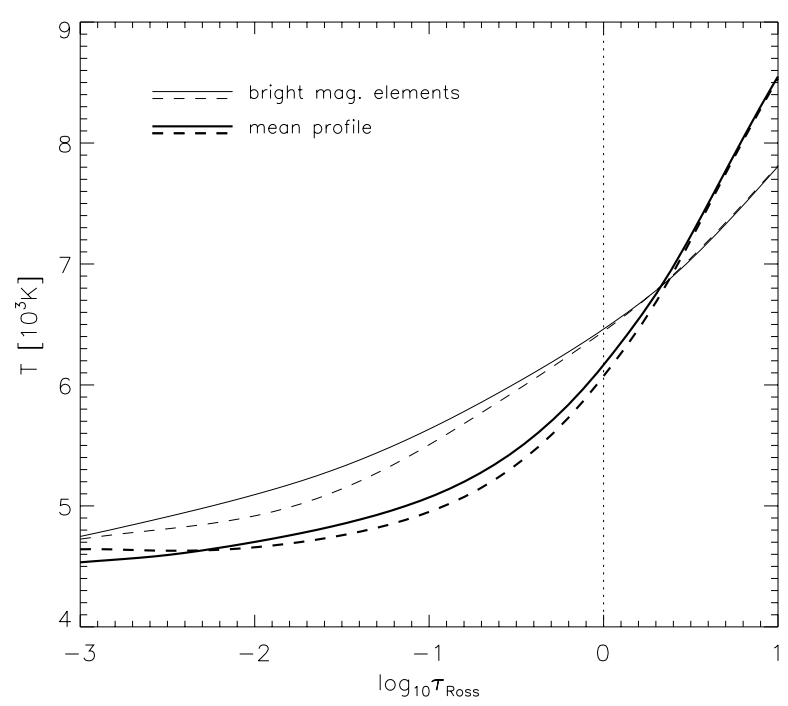

Fig. 10. Thin lines: vertical temperature profiles inside magnetic structures with above-average brightness. Bold lines: mean temperature profiles of the whole simulated domain. Solid lines correspond to the non-grey simulation run, dashed ones to the grey run. photosphere. In the grey case, the Rosseland mean underestimates the strength of absorbers and does not allow an adequate modelling of the smoothing effects of the radiative transfer. The difference is particularly pronounced in magnetic field concentrations.

- The non-grey radiative transfer enhances the effect of radiative illumination in magnetic flux concentrations.

- The differences in the temperature structure affect the appearance of the simulations in brightness maps: the rms intensity contrast is reduced to $15 \%$ in the non-grey case compared with $18 \%$ in the grey case. This reduction of the overall contrast leads to a contrast enhancement of bright magnetic elements.

Based on these results we conclude that grey calculations yield a sufficiently accurate modelling of the radiative transfer for simulations which focus on magnetoconvective processes at the surface and below. If results of numerical simulations are intended to be used as basis for spectral diagnostics, an adequate representation of the temperature structure in the middle and upper parts of the photosphere, particularly inside 
magnetic field concentrations, requires a non-grey treatment of the radiative transfer.

Acknowledgements. I would like to thank Manfred Schüssler for many helpful comments and fruitful discussions. I am grateful to Fausto Cattaneo, Thierry Emonet and Timur Linde from the University of Chicago for their hospitality during a stay at the UofC and for their contributions to the development of the MURaM code. This work was supported by the Max-Planck-Institute for Aeronomy through a doctoral stipend and by the DFG through grant SCHU 500/7. Financial support by the Max-Planck-Institute for Aeronomy during a working visit at the University of Chicago is gratefully acknowledged.

\section{References}

Deinzer, W., Hensler, G., Schüssler, M., \& Weisshaar, E. 1984, A\&A, 139,435

Knölker, M., Grossmann-Doerth, U., Schüssler, M., \& Weisshaar, E. 1991, Adv. Space Res., 11, 285
Kurucz, R. 1993, ATLAS9 Stellar Atmosphere Programs and 2 km/s grid., Kurucz CD-ROM No. 13., Cambridge, Mass., Smithsonian Astrophysical Observatory

Ludwig, H. G. 1992, Ph.D. Thesis, Universität Kiel

Ludwig, H.-G., Jordan, S., \& Steffen, M. 1994, A\&A, 284, 105

Mihalas, D. 1967, ApJ, 149, 169

Nordlund, A. 1982, A\&A, 107, 1

Schüssler, M. 2003, in Third International Workshop on Solar Polarization, ed. J. Trujillo Bueno, \& J. Sánchez Almeida, ASP Conf. Ser., in press

Sneden, C., Johnson, H. R., \& Krupp, B. M. 1976, ApJ, 204, 281

Spruit, H. C. 1976, Sol. Phys., 50, 269

Spruit, H. C. 1977, Sol. Phys., 55, 3

Strom, S. E., \& Kurucz, R. 1966, AJ, 71, 181

Vögler, A. 2003, Ph.D. Thesis, Göttingen University

Vögler, A., \& Schüssler, M. 2003, Astron. Nachr., 324, 399

Vögler, A., Shelyag, S., Schüssler, M., et al. 2003, in Modelling of Stellar Atmospheres, ed. N. E. Piskunov, W. W. Weiss, \& D. F. Gray, IAU Symp., 210, ASP Conf. Ser., 157

Vögler, A., Bruls, J. H. M. J., \& Schüssler, M. 2004, A\&A, 421, 741 